

\title{
Elaboración de briquetas a partir de residuos de aserrín aglutinados con almidón de maíz y su posible aplicación como aislante térmico
}

\author{
Preparation of briquettes from sawdust waste agglutinated \\ with corn starch and and its possible application as a \\ thermal insulator
}

\author{
Leixi Viviana García-Escalona \\ Universidad de Los Andes, Venezuela \\ Ingeniero Forestal. \\ Pedro Rafael Benítez-Díaz \\ Universidad de Los Andes, Venezuela \\ Licenciado en Química, MSc. Ciencias Médicas Fundamentales. \\ Styles Will Valero \\ Universidad de Los Andes, Venezuela \\ Ingeniero Forestal, MSc. Tecnología de Productos Forestales. \\ Ilvania Gutiérrez-Gotera \\ Universidad de Los Andes, Venezuela \\ Ingeniero Forestal, MSc. Tecnología de Productos Forestales.
}

Recibido: 24 de marzo 2018

Aceptado: 25 de setiembre 2018

\section{Resumen}

Se desarrolló una metodología que representa una opción en el aprovechamiento sostenible de residuos de aserrín de Pinus caribaea var. honduresis. Se elaboraron dos formulaciones de briquetas aglutinadas con almidón de maíz: 25/75 almidón/aserrín (lote A) y 36/64 almidón/aserrín (lote B). Los mejores resultados se obtuvieron con las briquetas del lote A: 45,2 $\pm 0,4 \mathrm{~mm}$ diámetro y $101 \pm 3 \mathrm{~mm}$ longitud; friabilidad < $1 \%$; humedad $9,4 \pm 0,5 \%$; densidad $0,45 \pm 0,01 \mathrm{~g} / \mathrm{ml}$; resistencia al aplastamiento $4,5 \times 10^{4} \pm 0,7 \times 10^{4}$ $\mathrm{kg} / \mathrm{m}^{2}$; poder calorífico $20,799 \mathrm{~kJ} / \mathrm{kg}$. Se estimó la capacidad de aislamiento térmico a 41,6 $\pm 0,4{ }^{\circ} \mathrm{C}$ y $57,3 \pm 0,1^{\circ} \mathrm{C}$; en el primer caso, la superficie de las briquetas se mantuvo en $29,0 \pm 0,4^{\circ} \mathrm{C}$ y en el segundo, en $34,7 \pm 0,8^{\circ} \mathrm{C}$, exhibiendo características térmicas similares a las del pino caribe. Las propiedades físico-mecánicas, físico-químicas y comportamiento térmico del material indican que podría tener utilidad como aislante térmico, representando una alternativa frente a su utilización como recurso energético. Iniciativas de este tipo contribuirían a una mayor sostenibilidad de la actividad forestal debido a que retarda la devolución a la atmósfera del $\mathrm{CO}_{2}$ fijado en los procesos de producción forestal. 
Palabras clave:

Briquetas, aislante térmico, ingeniería verde, industria forestal.

\begin{abstract}
A methodology was developed as an option for the sustainable use of sawdust residues of Pinus caribaea var. honduresis. Two briquette formulations agglutinated with cornstarch were prepared: 25/75 starch/sawdust (lot A) and 36/64 starch / sawdust (lot B). Therefore, the best results were obtained with the briquettes of lot A: $45.2 \pm 0.4 \mathrm{~mm}$ diameter and $101 \pm 3 \mathrm{~mm}$ length; friability < $1 \%$; humidity $9.4 \pm 0.5 \%$; density $0.45 \pm 0.01 \mathrm{~g} / \mathrm{ml}$; crush resistance $4.5 \times 10^{4} \pm 0.7 \times 10^{4} \mathrm{~kg} / \mathrm{m}^{2}$; calorific value $20.799 \mathrm{~kJ} / \mathrm{kg}$. The thermal insulation capacity was estimated at $41.6 \pm 0.4{ }^{\circ} \mathrm{C}$ and $57.3 \pm 0.1{ }^{\circ} \mathrm{C}$; in the first case, the surface of the briquettes remained at $29.0 \pm 0.4{ }^{\circ} \mathrm{C}$ and in the second, at $34.7 \pm 0.8^{\circ} \mathrm{C}$, exhibiting thermal characteristics similar to those of the caribbean pine. The physical-mechanical, physical-chemical and thermal properties of the material indicate that it could have utility as a thermal insulator, representing an alternative to its use as an energy resource. Initiatives of this type would contribute to a greater sustainability of the forestry activity, because it delays the return to the atmosphere of $\mathrm{CO}_{2}$ fixed in the forest production processes.
\end{abstract}

Keywords:

Briquettes, thermal insulation, green engineering, forestry industry. 


\section{INTRODUCCIÓN}

A partir de que la Organización de las Naciones Unidas (ONU) estableciera el desarrollo sostenible como principio rector para el desarrollo mundial a largo plazo, surgió la necesidad de que todas las actividades productivas humanas se enmarcaran dentro de este paradigma y el sector forestal no escapó a ello (Organización de las Naciones Unidas [ONU], 2010). Dentro de este contexto, la actividad forestal juega un papel de suma importancia debido a que los bosques y la biomasa forestal funcionan como sumideros y reservorios de gases efecto invernadero (Intergovernmental Panel on Climate Change [IPCC], 2015). Para adecuarse a los retos impuestos por el desarrollo sostenible, el sector forestal ha tenido que introducir innovaciones importantes en el aprovechamiento de los recursos forestales y la industria de transformación de la madera, y, sobre todo, en el aprovechamiento de los residuos que esta actividad genera (Avilés y López, 2015).

La industria de la madera constituye una de las más importantes fuentes generadoras de residuos, representando un severo problema ambiental, esto ocurre a través de todos los procesos relacionados con ella, desde la explotación de los bosques y transformación primaria, hasta la obtención de toda la gama de productos finales (Rojo, Jaso y Velásquez, 2003; Concepción, Chonillo, Lorenzo y Morales, 2016). Según la Organización de las Naciones Unidas para la Alimentación y la Agricultura (Food and Agriculture Organization of the United Nations [FAO], 2017), el $60 \%$ del árbol talado se queda en el bosque, y entre el $45 \%$ y $55 \%$ de las trozas que entran en los aserraderos o fábricas de contrachapados se convierten en residuos, a estos se suman lo aportado por la industria de segunda transformación y la industria papelera (Velázquez, 2006; Lucas, Peso, Rodríguez y Prieto, 2012). Aserrín, virutas, despuntes y costaneras, entre otros, generalmente son dispuestos en el medio sin adoptar medidas de control, prevención o mitigación, siendo almacenados en grandes cerros, quemados en calderas o en el peor de los casos, quemados a cielo abierto. De esta forma, se convierten en focos de contaminación, afectando en primer término a las poblaciones y cuerpos de agua adyacentes a las zonas de explotación y transformación; y en segundo término, a las poblaciones aguas abajo (López, et al., 2016). Los problemas generados por la producción de residuos forestales pudieran ser solventados o disminuidos al dárseles un mayor valor agregado o eficiencia energética, contribuyendo a la conservación del ambiente y a disminuir las emisiones de gases efecto invernadero (Concepción et al., 2016).

En la búsqueda de dar solución a la problemática planteada, se viene considerando, desde hace mucho tiempo, la utilización de la biomasa forestal residual como materia prima para la obtención de energía. A este respecto, Avilés y López (2015) indican que esta actividad ha alcanzado tal avance tecnológico, que hoy en día representa una de las alternativas energéticas más baratas y sostenibles para muchos países en vías de desarrollo, ayudando también a la solución a los problemas socioeconómicos y ambientales que se generan por el limitado acceso y alto costo de la energía (López et al., 2016).

Particularmente en Venezuela, el aserrín es visto como un desecho y es dispuesto inadecuadamente al entorno, convirtiéndose en un serio problema ambiental. Ante esto, la industria forestal venezolana debe plantearse el reto de cambiar esta perspectiva, 
desarrollando nuevos productos y aplicaciones que permitan emplear como materia prima lo que hasta ahora se considera un desperdicio, como una forma de contribuir a la solución del problema y a dar mayor sostenibilidad a su actividad productiva. Algunos autores (Smith y Bracho, 2011), consideran que en Venezuela la utilización de la biomasa residual forestal como biocombustible podría representar una vía para resolver problemas energéticos, de saneamiento ambiental y reducción de emisiones de $\mathrm{CO}_{2}$. Sin embargo, la realidad es que esta alternativa es poco viable debido al muy bajo costo del combustible fósil, el cual es subsidiado por el Estado Venezolano (Posso, 2004; Posso y Zambrano, 2014). Por las razones antes expuestas, se ha planteado la necesidad de proponer una alternativa sostenible para aprovechar los residuos de aserrín que sea diferente a su utilización como recurso energético, que además de prolongar el tiempo de devolución a la atmósfera del $\mathrm{CO}_{2}$ fijado en el proceso biológico de producción forestal, confiera mayor valor agregado a un material que es considerado un desecho (Medina, Palma y Castro, 2016).

El Pinus caribaea var. hondurensis (pino caribe), ampliamente distribuido en el continente americano (Greaves, Marín, Visaez y Hernández, 2015), representa la mayor producción de madera en rolas en Venezuela y es la madera de menos valor económico disponible para las carpinterías (Loreto, Molina, Vivas, Lugo y Conti, 2000; Reyes, Molina, Valero, Molina y Betancourt, 2012), por lo tanto, constituye también la mayor proporción de los residuos de aserrín. Greaves et al. (2015), plantean que la infraestructura y experiencia que posee el país para la propagación del pino caribe son recursos que pudieran utilizarse en la recuperación de áreas desérticas del planeta debido a que funcionancomo sumideros y reservorios de gases efecto invernadero.

El presente trabajo tiene como objetivo desarrollar una opción metodológica que contribuya al aprovechamiento sostenible de los residuos de aserrín, proponiendo una aplicación diferente a la bioenergética; explorando, a su vez, la posibilidad de su utilización como aislante térmico. Para ello, se plantea la elaboración de un material en forma de briqueta cilíndrica por su facilidad de elaboración y manejo, empleando un procedimiento e instrumentación sencillos, diseñados a partir insumos de fácil adquisición en el mercado. Debido a que el estudio está enmarcado dentro de los lineamientos de la Química e Ingeniería Verde (García y Dobado, 2008; Gómez, 2008), se utiliza almidón de maíz (Zea mays L.) como sustancia aglutinante, lo cual permite la obtención de un producto biodegradable, evitando el impacto ambiental ocasionado por las resinas sintéticas comúnmente empleadas en la fabricación de tableros y otros productos aglomerados. Como parte de la caracterización del producto obtenido se evaluarán algunas propiedades físico-mecánicas y físico-químicas.

\section{MATERIALES Y MÉTODOS}

\subsection{Materiales y equipos}

Se utilizó almidón de maíz para consumo humano, marca Maizina Americana, Alfonso Rivas \& Cía. Por su parte, el aserrín de pino caribe se obtuvo de los residuos 
generados en el aserradero y carpintería del Laboratorio Nacional de Productos Forestales (LNPF) de la Universidad de Los Andes, Mérida, Venezuela. Para la elaboración de las briquetas se utilizaron moldes constituidos por tubos de cloruro de polivinilo (PVC) de $5,08 \mathrm{~cm}$ de diámetro interno y $15 \mathrm{~cm}$ de longitud, los cuales fueron cerrados en sus extremos con tapas removibles del mismo material. Para la compresión de las briquetas se empleó un embolo de metal de $680 \mathrm{~g}$.

Los equipos utilizados fueron: horno de microondas doméstico, marca Panasonic modelo $\mathrm{N}^{\circ} \mathrm{NN}-\mathrm{ST} 678 \mathrm{~S}, 60 \mathrm{~Hz}, 12,7 \mathrm{~A}, 120 \mathrm{~V}$. Estufa de convección forzada marca Germany GLOBE modelo DOD - A 053 Drying Oven. Prensa universal para ensayos marca WOLPERT modelo U5, máxima capacidad 5 Ton. Circulador termostático marca Colora modelo ULTRA-THERMOSTAT NB5.Termómetro infrarrojo digital marca FLUKE, modelo 566/568.

\subsection{Metodología}

\subsubsection{Elaboración de briquetas}

A partir de ensayos preliminares se logró establecer un procedimiento para la elaboración de las briquetas cilíndricas, que comprende los pasos que se describen a continuación.

\subsubsection{Acondicionamiento del aserrín y preparación del engrudo de almidón}

El aserrín fue molido hasta obtener un tamaño de partícula de 0,10 $\pm 0,04 \mathrm{~mm}$, lo cual se considera harina o polvo de aserrín (FAO, 2004). Posteriormente, fue llevado a un contenido de humedad $(\mathrm{CH})<1 \%$ utilizando horno de microondas (HMO), adaptando el procedimiento desarrollado por Benítez y Gutiérrez (2012).

Para la preparación del engrudo se hidrataron $30 \mathrm{~g}$ de almidón en $60 \mathrm{ml}$ de agua destilada y aparte se calentaron $120 \mathrm{ml}$ de agua en $\mathrm{HMO}$ por 2 min (máxima potencia). Luego, se añadió el agua hirviendo al almidón hidratado y se mezcló evitando la formación de grumos, se terminó la preparación calentando la mezcla en HMO (máxima potencia) con 1 pulso de $30 \mathrm{~s}$, seguido de 3 pulsos de $15 \mathrm{~s}$, agitando entre pulsos hasta obtener la consistencia de engrudo. Posteriormente, se dejó en reposo hasta llegar a temperatura ambiente.

\subsubsection{Formulación y moldeado de las briquetas}

Se elaboraron dos lotes de briquetas, para el lote A se mezclaron 122,5 g de engrudo y 52,5 g de aserrín (70\% aglutinante / $30 \%$ aserrín); para el lote B se mezclaron 140,0 g de engrudo y $35,0 \mathrm{~g}$ de aserrín (80 \% aglutinante / $20 \%$ aserrín). Después de que los componentes estuvieron bien integrados, la mezcla fue sometida a 3 pulsos de $30 \mathrm{~s}$ en el HMO, con intervalos de enfriamiento de 1 min entre cada pulso, con la finalidad de eliminar parte de la humedad. Luego, se dejó reposar hasta llegar a temperatura ambiente y paso seguido se procedió a rellenar los moldes, previamente recubiertos con aceite vegetal. Se forzó la compactación de la briqueta, empleando el embolo metálico 
y ejerciendo presión con el brazo humano hasta una altura aproximada de $10 \mathrm{~cm}$. Las briquetas del lote A fueron desmoldadas después de $48 \mathrm{~h}$ y las del lote B fueron desmoldadas trascurridas $72 \mathrm{~h}$.

Tabla 1. Esquema de calentamiento en el HMO para el secado de las briquetas del lote A en grupos de 10.

\begin{tabular}{cccc}
\hline Paso & Tiempo HMO & $\begin{array}{c}\text { Tiempo de enfriamiento } \\
\text { al ambiente }\end{array}$ & Pesada (al 0,1g) \\
\hline 0 & 0 & 0 & Pesar \\
1 & 1 min $30 \mathrm{~s}$ & 2 min & \\
2 & 1 min $30 \mathrm{~s}$ & 2 min & \\
3 & 1 min $30 \mathrm{~s}$ & 2 min & \\
4 & 1 min $30 \mathrm{~s}$ & 2 min & Pesar \\
5 & 1 min $30 \mathrm{~s}$ & 2 min & Pesar \\
6 & 1 min $30 \mathrm{~s}$ & 20 min & Hasta peso constante (PBs) \\
7 & 1 min & 15 min & \\
8 & Repetir & Repetir & \\
& & & \\
\hline
\end{tabular}

HMO: horno microondas; PBh: peso de la briqueta húmeda; PBs: peso de la briqueta seca.

Tabla 2. Esquema de calentamiento en el HMO para el secado de las briquetas del lote $\mathrm{B}$ en grupos de 10

\begin{tabular}{cccc}
\hline Paso & Tiempo HMO & $\begin{array}{c}\text { Tiempo de enfriamiento } \\
\text { al ambiente }\end{array}$ & Pesada (al 0,1g) \\
\hline 0 & 0 & 0 & Pesar (PBh) \\
1 & 2 min & 2 min & \\
2 & 2 min & 2 min & \\
3 & 2 min & 2 min & \\
4 & 1 min $30 \mathrm{~s}$ & 2 min & \\
5 & 1 min $30 \mathrm{~s}$ & 2 min & \\
6 & 1 min $30 \mathrm{~s}$ & 2 min & Pesar \\
7 & 1 min $30 \mathrm{~s}$ & 2 min & Pesar \\
8 & 1 min $30 \mathrm{~s}$ & 20 min & Hasta peso constante (PBs) \\
9 & 1 min & 15 min & Repetir \\
10 & Repetir & &
\end{tabular}

HMO: horno microondas; $\mathrm{PBh}$ : pesode labriqueta húmeda; $\mathrm{PBs}$ : pesode labriqueta seca. 


\subsubsection{Secado de las briquetas}

El proceso de secado se realizó en dos etapas; en la primera, las briquetas fueron introducidas en una estufa de convección forzada a $45^{\circ} \mathrm{C}$ durante $48 \mathrm{~h}$, con este procedimiento se garantiza la integridad física de las briquetas. En la segunda etapa se diseñó un esquema de secado en $\mathrm{HMO}$, las briquetas fueron secadas en grupos de 10 hasta llegar a peso constante, en las Tablas 1 y 2 se muestra el procedimiento para cada lote, esta metodología permitió acelerar el proceso de secado y eliminar toda el agua empleada en el proceso. Posteriormente, las briquetas se mantuvieron en el cuarto de acondicionamiento (humedad de $65 \pm 2 \%$ y temperatura de $20 \pm 1{ }^{\circ} \mathrm{C}$ ) hasta alcanzar el equilibrio higroscópico, lo cual se monitoreó haciendo medidas diarias hasta obtener peso constante. Culminado este procedimiento, se procedió a la determinación del contenido de humedad y a realizar las pruebas correspondientes.

\subsubsection{Determinación del $\mathrm{CH}$}

Para la determinación del $\mathrm{CH}$ se tomó como el peso de la briqueta seca (PBs) obtenido al concluir la segunda etapa del secado (sección 2.2.1.3) y como el peso de la briqueta húmeda ( $\mathrm{PBh})$, el alcanzado después de llegar al equilibrio higroscópico en el cuarto de acondicionamiento. El CH se calculó según la ecuación 1.

$$
C H=\frac{P B h-P B s}{P B S} \times 100
$$

Donde:

$\mathrm{CH}$ Contenido de humedad (\%)

$\mathrm{PBh} \quad$ Peso de la briqueta húmeda $(\mathrm{g})$

PBs Peso de la briqueta seca $(\mathrm{g})$

\subsubsection{Determinación de Densidad}

Para determinar su densidad, las briquetas fueron pesadas una vez que alcanzaron el equilibrio higroscópico en el cuarto de acondicionamiento y luego, se estimó su volumen por inmersión o desplazamiento de agua. El ensayo se realizó con 20 briquetas y la densidad se reportó en $\mathrm{g} / \mathrm{ml}$.

\subsubsection{Determinación del Índice de Friabilidad}

Para evaluar la friabilidad se utilizó la combinación de dos métodos, el primero reseñado en otros trabajos (Soto y Núñez, 2008; Fonseca y Tierra, 2011; Ordóñez, 2015). Este consiste en dejar caer las briquetas, orientadas verticalmente, cinco veces 
sobre un suelo cerámico desde $1 \mathrm{~m}$ de altura. El segundo método, fundamentado en la Farmacopea Argentina (Administración Nacional de Medicamentos, Alimentos y Tecnología Médica [ANMAT], 2003), consiste en determinar el índice de friabilidad (IFr) como el porcentaje de pérdida de peso después de realizada la prueba, según la ecuación 2. Adicionalmente, con la finalidad de complementar la caracterización de las briquetas, se realizó una prueba de friabilidad dejando caer las briquetas una vez desde $5 \mathrm{~m}$ de altura sobre un suelo cerámico, haciendo el cálculo de la misma manera.

$$
I F r=\frac{P i-P f}{P i} \times 100
$$

Donde:

IFr Índice de friabilidad (\%)

Pi Peso inicial de la briqueta $(\mathrm{g})$

Pf Peso final de la briqueta $(\mathrm{g})$

\subsubsection{Determinación de la resistencia al aplastamiento}

El ensayo fue realizado en las instalaciones de la Sección de Ensayos del LNPF, de acuerdo con el siguiente procedimiento: la briqueta, mantenidas hasta el momento de la prueba en el cuarto de acondicionamiento, se colocó longitudinalmente entre dos superficies planas de acero de $10 \mathrm{~cm}$ x $10 \mathrm{~cm}$, luego se le aplicó la carga de compresión a una velocidad de $5 \mathrm{~kg} / \mathrm{s}$ y se leyó la fuerza (carga máxima, $\mathrm{kg}$ ) de compresión en el momento de la destrucción de la briqueta. Debido a que el ensayo es destructivo, se realizó sobre una muestra de 18 briquetas en cada lote. La resistencia al aplastamiento $\left(\mathrm{RA}, \mathrm{kg} / \mathrm{m}^{2}\right.$ ), se obtuvo dividiendo la lectura entre la superficie de la briqueta donde se aplicó la fuerza.

\subsubsection{Estimación de la capacidad de aislamiento térmico}

Para cada lote se seleccionaron al azar un conjunto de briquetas, las cuales fueron cortadas transversalmente para obtener discos de $2 \mathrm{~cm}$ de espesor. Los discos obtenidos fueron pegados en dos caras (una para cada lote) de un recipiente de vidrio cuadrado, utilizando como adhesivo una delgada línea de silicona sobre el borde de los discos y dejando curar durante 24 h. Para tener un término de comparación, en la tercera cara del recipiente y utilizando el mismo procedimiento, se colocaron discos de madera de pino caribe del mismo espesor $(2 \mathrm{~cm})$, orientados en sentido perpendicular a la fibra.

Posteriormente, el recipiente fue llenado con agua destilada que se hizo pasar a través de un circulador termostático. Se realizaron dos experimentos a temperatura 
ambiente $\left(23^{\circ} \mathrm{C}\right)$, en el primero el agua del baño se mantuvo a $43,5^{\circ} \mathrm{C}$ y en el segundo a $60,5^{\circ} \mathrm{C}$. En ambos casos, se tomaron lecturas de la temperatura cada 10 min durante $2 \mathrm{~h}$, tanto en las caras descubiertas del recipiente de vidrio (considerada la temperatura control) como sobre la superficie expuesta al aire de los discos de las briquetas y madera de pino caribe.

\subsubsection{Reporte de Resultados}

Todos los resultados fueron reportados como el promedio más o menos la desviación estándar ( $\overline{\boldsymbol{x}} \pm \boldsymbol{D S})$. Las diferencias estadísticamente significativas se determinaron a través de un análisis de varianza (ANOVA de una vía) empleando el programa GraphPadInStat.

\section{RESULTADOS}

Las briquetas se elaboraron con engrudo de almidón de maíz preparado al 14,3\% en agua, con una formulación 30/70 aserrín/engrudo para el lote A y 20/80 aserrín/engrudo para el lote B. Una vez eliminada el agua de proceso, y después que alcanzaron el equilibrio higroscópico, resultaron con una composición final de 75,0/25,0 aserrín/almidón para el lote A y 63,6/36,4 aserrín/almidón para el lote B.

Tabla 3. Propiedades físico-mecánicas y fisicoquímicas de las briquetas.

\begin{tabular}{ccccc}
\hline & Lote A & Lote B & $\mathrm{n}$ & $\mathrm{p}$ \\
\hline Diámetro & $45,2 \pm 0,4 \mathrm{~mm}$ & $44,1 \pm 0,8 \mathrm{~mm}$ & 50 & $>0,05$ \\
Longitud & $101 \pm 3 \mathrm{~mm}$ & $84 \pm 4 \mathrm{~mm}$ & 50 & $<0,001$ \\
PBh & $70 \pm 1 \mathrm{~g}$ & $52 \pm 3 \mathrm{~g}$ & 50 & $<0,001$ \\
PBs & $64 \pm 1 \mathrm{~g}$ & $48 \pm 3 \mathrm{~g}$ & 50 & $<0,001$ \\
CH & $9,4 \pm 0,5 \%$ & $8 \pm 2 \%$ & 50 & $<0,01$ \\
Vi & $155 \pm 3 \mathrm{ml}$ & $100 \pm 3 \mathrm{ml}$ & 20 & $<0,001$ \\
Densidad & $0,45 \pm 0,01 \mathrm{~g} / \mathrm{ml}$ & $0,51 \pm 0,01 \mathrm{~g} / \mathrm{ml}$ & 20 & $>0,05$ \\
IFr (1m*5) & $<1 \%$ & $<1 \%$ & 10 & $\mathrm{~ns}$ \\
IFr (5m*1) & $<1 \%$ & $<1 \%$ & 10 & $\mathrm{~ns}$ \\
RA & $4,5 \times 10^{4} \pm 0,7 \times 10^{4} \mathrm{~kg} / \mathrm{m}^{2}$ & $6,5 \times 10^{2} \pm 0,2 \times 10^{2} \mathrm{~kg} / \mathrm{m}^{2}$ & 18 & $<0,0001$ \\
\hline
\end{tabular}

PBh: peso de la briqueta húmeda; PBs: peso de la briqueta seca; $\mathrm{CH}$ : contenido de humedad; Vi: volumen por inmersión; IFr: índice de friabilidad; RA: resistencia al aplastamiento; n: número de muestras; p: significación estadística; ns: no significativo.

En la Tabla 3 se muestran los resultados obtenidos de la evaluación de las propiedades físico-mecánicas y físico-químicas de las briquetas. El diámetro de ambos lotes es prácticamente el mismo, como es de esperarse por el tipo de molde 
empleado. Por el contrario, la longitud del lote B es alrededor de $17 \%$ menor que las del lote A, siendo estadiferencia estadísticamente significativa. También se observaron diferencias significativas entre los lotes para el PBh y PBs, en ambos casos el peso del lote A fue 1,3 veces mayor a los del lote $\mathrm{B}$. En cuanto al $\mathrm{CH}$, al igual que en los casos anteriores sigue siendo mayor el del lote A, aunque la diferencia es menos significativa debido a la mayor variabilidad mostrada por el lote $\mathrm{B}(\mathrm{CV}=27,1 \%)$. El volumen por inmersión (Vi) del lote A es alrededor de 1,5 veces mayor que el del lote $\mathrm{B}$, siendo esta diferencia estadísticamente significativa, resultando que la densidad del lote B es aproximadamente $13 \%$ mayor a la del lote A, aunque esta diferencia no es estadísticamente significativa debido a la mayor variabilidad del lote $\mathrm{B}(\mathrm{CV}=$ $25 \%$ ). Hay que destacar que en el interior de las briquetas del lote B se observaron espacios vacíos de forma irregular, como se observa en el corte transversal mostrado en la Figura 3.

El IFr para ambos lotes fue menor al $1 \%$, tanto para la prueba de cinco lanzamientos desde un metro de altura como para la prueba de un lanzamiento desde $5 \mathrm{~m}$. En cuanto a la RA, el promedio mostrado por el lote A es dos órdenes de magnitud mayor que el del lote B. Lo anterior representa una diferencia muy significativa desde el punto de vista estadístico.

En las Figuras 1 y 2 se muestran los gráficos correspondientes a los resultados obtenidos para la estimación de la capacidad de aislamiento térmico de las briquetas del lote A y B. En la primera prueba (Figura 1), se observa que a partir del minuto 20 se estabilizan todas las temperaturas y se mantiene una diferencia alrededor de $12{ }^{\circ} \mathrm{C}$ $(\sim 29 \%)$ entre el control $\left(41,6 \pm 0,4^{\circ} \mathrm{C}\right)$ y la superficie de las briquetas de ambos lotes (lote A $29,4 \pm 0,4^{\circ} \mathrm{C}$ y lote B $30,0 \pm 0,5^{\circ} \mathrm{C}$ ), lo cual es estadísticamente significativo $(\mathrm{p}<0,001)$. La diferencia de temperatura entre ambos lotes es muy pequeña $(0,6$ $\left.{ }^{\circ} \mathrm{C}\right)$ y poco significativa $(\mathrm{p}<0,01)$. Por otra parte, la superficie de madera de pino caribe se mantuvo $14,7^{\circ} \mathrm{C}(\sim 35 \%)$ por debajo del control $(\mathrm{p}<0,001)$. Finalmente, la temperatura del pino caribe estuvo por debajo de ambos lotes de briquetas, con respecto al lote $\mathrm{A}$ la diferencia fue de $2,1^{\circ} \mathrm{C}(\sim 7 \%, \mathrm{p}<0,001)$ y para el lote $\mathrm{B}$ de $3,1{ }^{\circ} \mathrm{C}(\sim 10 \%, \mathrm{p}<0,001)$.

Para la segunda prueba de resistencia térmica (Figura 2), el baño termostático se fijó en $60,5^{\circ} \mathrm{C}$ y las temperaturas se estabilizaron a partir del minuto 40 . Desde ese momento, el control se mantuvo en $57,3 \pm 0,1^{\circ} \mathrm{C}$ y la superficie de las briquetas de ambos lotes $22^{\circ} \mathrm{C}$ por debajo $(\sim 38 \%)$, correspondiendo a un resultado estadísticamente significativo $(\mathrm{p}<0,001)$. Al igual que en la primera prueba, la diferencia de entre los lotes es muy pequeña $\left(0,8^{\circ} \mathrm{C}\right)$ y poco significativa $(\mathrm{p}<0,01)$. En este caso, la temperatura de la madera de pino caribe estuvo $24,5{ }^{\circ} \mathrm{C}$ por debajo del control $(\sim$ $43 \%, \mathrm{p}<0,001), 1,9^{\circ} \mathrm{C}$ menor que el lote $\mathrm{A}(\sim 5 \%, \mathrm{p}<0,001)$ y $2,7^{\circ} \mathrm{C}$ más baja que el lote $\mathrm{B}(\sim 8 \%, \mathrm{p}<0,001)$.

Es importante destacar que para la segunda prueba la temperatura control se incrementó $15,7^{\circ} \mathrm{C}$ y la de la superficie de las briquetas expuesta al ambiente subió sólo $5,3{ }^{\circ} \mathrm{C}$ para el lote $\mathrm{A}(\mathrm{p}<0,001), 5,5^{\circ} \mathrm{C}$ para el lote $\mathrm{B}(\mathrm{p}<0,001)$ y la madera de pino caribe $5,9^{\circ} \mathrm{C}$. 


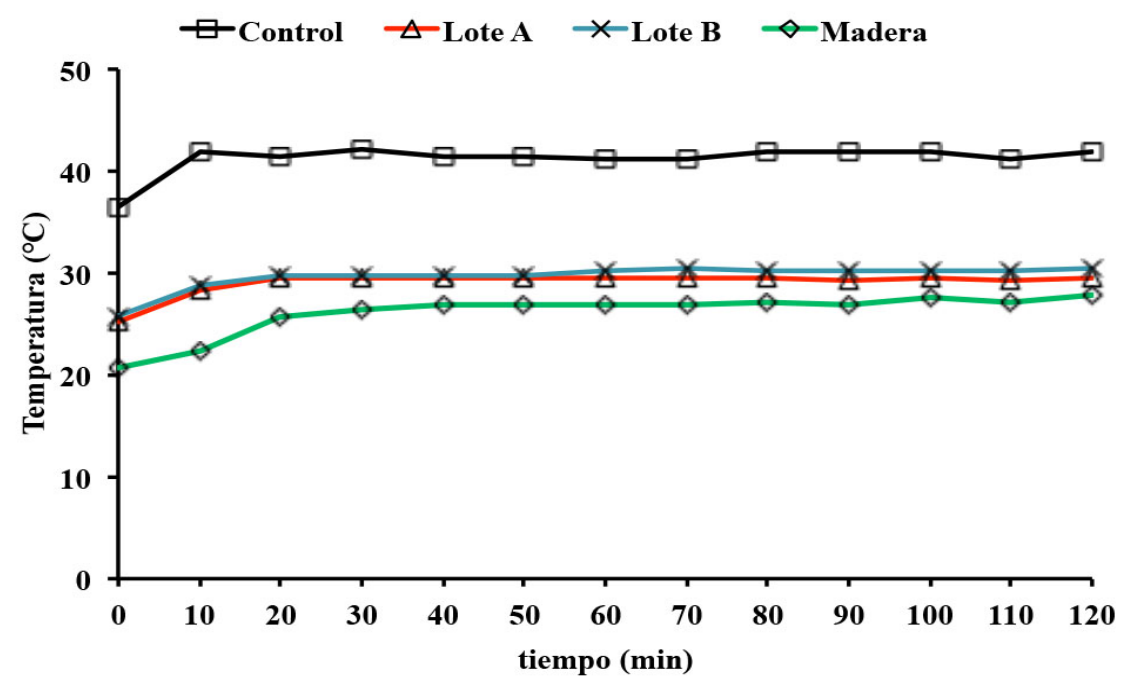

Figura 1. Primera prueba de aislamiento térmico realizada a temperatura ambiente $\left(23^{\circ} \mathrm{C}\right)$, el baño termostático se ajustó a $43,5^{\circ} \mathrm{C}$, a partir del minuto 20 la temperatura control (superficie del vidrio) se mantuvo en $41,6 \pm 0,4^{\circ} \mathrm{C}$, la superficie de la briqueta del lote A en $29,0 \pm 0,4{ }^{\circ} \mathrm{C}$, la del lote B $30,0 \pm 0,5^{\circ} \mathrm{C}$ y la madera de pino caribe en $27 \pm 1$.

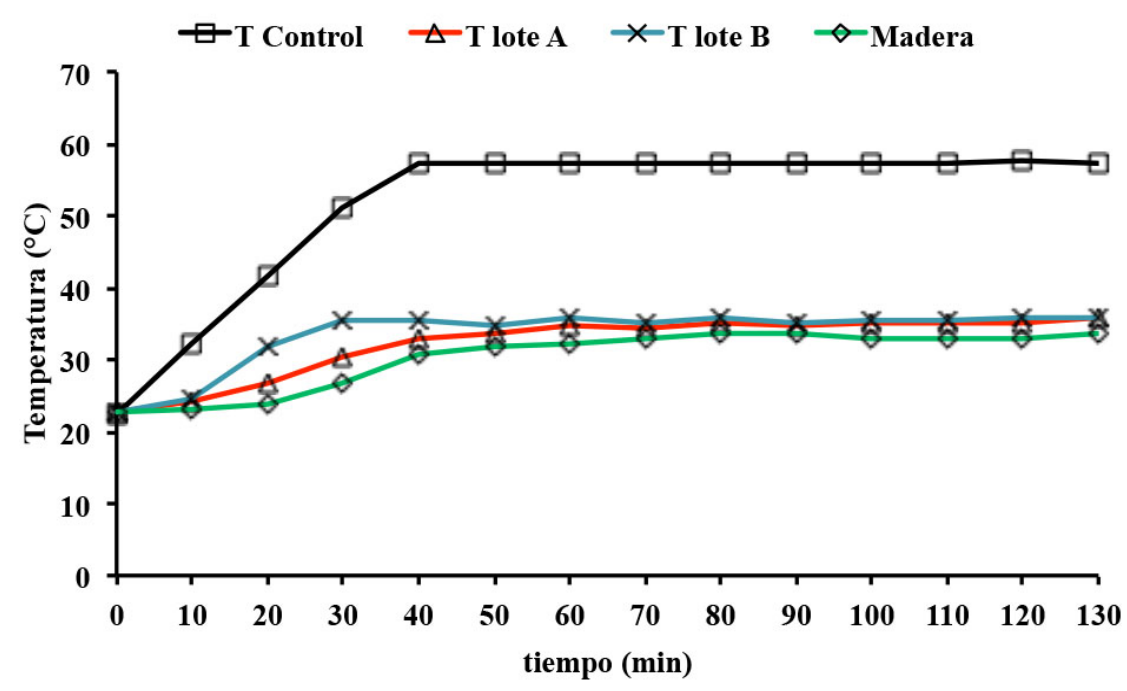

Figura 2. Segunda prueba de aislamiento térmico realizada a temperatura ambiente $\left(23^{\circ} \mathrm{C}\right)$, el baño termostático se ajustó a $60,5^{\circ} \mathrm{C}$, a partir del minuto 40 la temperatura control (superficie de vidrio) se mantuvo en $57,3 \pm 0,1^{\circ} \mathrm{C}$, la superficie de la briqueta del lote $\mathrm{A}$ en $34,7 \pm 0,8^{\circ} \mathrm{C}$, el lote B $35,5 \pm$ $0,3{ }^{\circ} \mathrm{C}$ y la madera de pino caribe en $32,8^{\circ} \mathrm{C}$. 


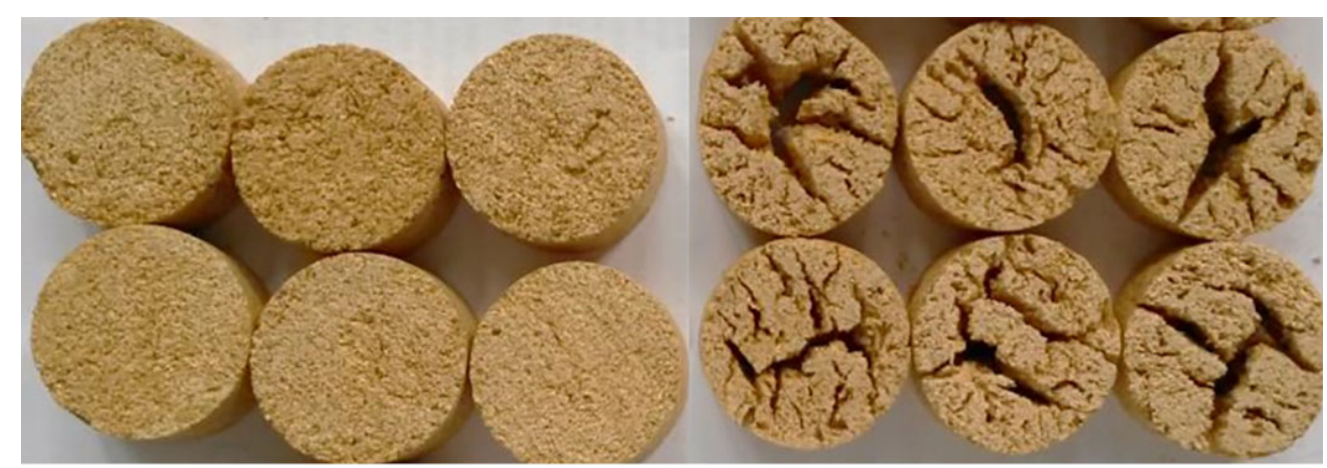

Lote $\mathrm{A}$

Lote B

Figura 3. Corte transversal de las briquetas. Lote A: se observa mejor compactación del material y un acabado uniforme; Lote B: Se observan espacios vacíos en el interior, menor compactación y apariencia no uniforme.

\section{DISCUSIÓN}

Comúnmente las briquetas son destinadas al uso bioenergético y existe una amplia diversidad en cuanto su forma y dimensiones, las cuales van a estar sujeta a los requerimientos y preferencias del fabricante. Aunque el objetivo del presente trabajo está fuera del área energética, se eligió elaborar un aglomerado en forma de briqueta cilíndrica debido a que presenta ventajas en cuanto a su fácil elaboración, además de que permite obtener un producto menos friable frente a la manipulación y el traslado (Fonseca y Tierra, 2011; Martínez, 2015; Gallipoliti, Martina, Corace, Aeberhardt y García, 2012).

El tamaño de partícula y $\mathrm{CH}$ de la materia prima que se utiliza para la elaboración de las briquetas tiene una importante influencia sobre las propiedades físico-mecánicas del producto final. En general, un menor tamaño de partícula y baja humedad favorecen una mayor interacción o fuerza de unión entre los componentes de la briqueta y por ende, se obtendrá un mejor acabado y mejores propiedades. Algunos autores que han trabajado en la elaboración de briquetas para bioenergía a partir de aserrín, califican como óptimo un tamaño de partícula inferior a $3 \mathrm{~mm}$ y un $\mathrm{CH}$ menor del $14 \%$ (Fonseca y Tierra, 2011; Gallipoliti et al., 2012; Tirado, 2015; FAO, 2014; Ordoñez, 2015). Para el presente trabajo se empleó aserrín con un tamaño de partícula de 0,14 $\pm 0,04 \mathrm{~mm}$ y CH inferior al $1 \%$, bajo estas condiciones se incrementó la superficie de interacción entre las partículas de aserrín y las moléculas de almidón, las cuales se encontraban desdobladas e hidratadas en el engrudo. Esto permitió explotar al máximo las similitudes estructurales entre estas últimas y los componentes moleculares del aserrín, principalmente celulosa y lignina, logrando una cohesión fuerte y estable (Pineda, Coral, Arciniegas, Rorales y Rodríguez, 2010; Agama, Juárez, Evangelista, Rosales y Bello, 2013; Hang et al., 2015).

Las dimensiones de las briquetas obtenidas (Tabla 3) se encuentran dentro de los parámetros comúnmente reportados en la literatura (Tirado, 2015; Fonseca y Tierra, 2011; 
Gallipoliti et al., 2012; Valderrama, Curo, Quispe, Llanto, y Gallo, 2007; Vera, 2014). Aunque los diámetros de las briquetas del lote A y B son similares, lo cual es de esperarse por el tipo de molde y procedimiento empleados, la longitud del lote B es significativamente menor que la del lote A. Esto es debido a que el menor porcentaje de aserrín que contiene el lote B permitió un mayor desplazamiento del émbolo durante el moldeado. Lo anterior llevó a una briqueta de menor tamaño pero menos compacta que la del lote $\mathrm{A}$, como se aprecia en la Figura. 3. Estas observaciones confirman que el aserrín es el componente más importante de la formulación y el que le confiere soporte estructural al material obtenido.

Debido a que el lote A tienen un mayor contenido de aserrín y mejor compactación que las del lote B, se podría inferir que las primeras deberían mostrar una densidad mayor, pero los resultados experimentales muestran lo contrario, las briquetas del lote $\mathrm{B}$ tienen una densidad aproximadamente $13 \%$ mayor que las del lote A, aunque esta diferencia es poco significativa (Tabla 3 ). Esto se debe a que a pesar de que el lote $\mathrm{B}$ tienen un peso promedio $26 \%$ menor que el lote A, la diferencia en volumen es aún mayor (aproximadamente $35 \%$ menos), como consecuencia de su disminución en su longitud. Por otra parte, es importante tomar en cuenta que en este trabajo se calculó la densidad aparente a partir de la masa de la briqueta y su volumen aparente determinado por inmersión (Lucas et al., 2012, Tirado, 2015), el cual incluye el volumen real más el volumen de los espacios vacíos que se observan en el corte transversal del lote B (Figura 3), por lo tanto su densidad real podría ser aún mayor que la reportada.

Al revisar la densidad del material obtenido, se encuentra que no cumple con la densificación de la biomasa que normalmente se requiere cuando se elaboran briquetas destinadas a uso energético (Fonseca y Tierra, 2011; Martínez, 2015; FAO, 2014), la densidad de las briquetas es inferior a la densidad de la madera de pino caribe (0,564 g/ml, seca al horno, según Reyes y col., 2012) de la cual proviene el aserrín utilizado en su elaboración. Como el propósito de la investigación no está dirigido hacia el aprovechamiento energético, no es indispensable cumplir con esta premisa, esto porque para la aplicación propuesta no se necesita la alta densidad requerida en los biocombustibles. Por el contrario, la baja densidad podría favorecer su utilización como aislante térmico porque atraparía más aire entre sus poros y adicionalmente, la carga sobre las paredes sería más baja permitiendo utilizar adhesivos menos resistentes (Peraza, Arriaga y Peraza, 2004). Adicionalmente, para obtener briquetas de elevada densidad es imprescindible utilizar maquinaria como prensas briqueteadoras o prensas hidráulicas que generen presiones de compactación mayores a $100 \mathrm{MPa}$, y un tipo de molde resistente a esas condiciones (FAO, 2014; Tirado, 2015; Fonseca y Tierra, 2011); en este caso se empleó la fuerza del brazo humano y moldes de PVC, cumpliendo con el objetivo de emplear una metodología e instrumentación sencillas.

Los resultados para el índice de friabilidad indican que las briquetas de ambos lotes tienen una alta resistencia al desmoronamiento, pudiendo ser manipuladas y transportadas sin que pierdan su integridad estructural (Fonseca y Tierra, 2011; Lucas et al., 2012; Martínez, 2015), siendo esta característica la consecuencia directa de la fuerte interacción entre el aserrín y el almidón utilizado como aglutinante.

Lucas et al. (2012), señalan que durante el proceso de manufactura de briquetas se debe conseguir un $\mathrm{CH}$ dentro del rango $8-10 \%$, por otra parte, FAO (2014) indica 
que el mismo se debe encontrar entre 6-8\%, y como se puede apreciar en la Tabla 3, el $\mathrm{CH}$ para ambos lotes se ubica dentro del rango esperado. Más aún, la metodología desarrollada en el presente trabajo arrojó mejores resultados que los encontrados por otros autores que reportan $\mathrm{CH}$ hasta de $12,3 \%$ y $11,1 \%$, para briquetas elaboradas a partir de aserrín de pino blanco aglutinado con almidón de yuca y cáscara de nuez sin aglutinante, respectivamente (Gallipoliti et al., 2012; Tirado, 2015). Además, se podría decir que el material obtenido tiene un $\mathrm{CH}$ por debajo del que se esperaría para la madera en las mismas condiciones, es decir en equilibrio higroscópico, lo cual podría ser provechoso para la aplicación propuesta (Arroyo, 2003).

Los valores de RA reportados (Tabla 3), se ubican alrededor de dos y cuatro órdenes de magnitud (lote A y B, respectivamente) por debajo de lo encontrado por otros autores para briquetas empleadas con fines energéticos $\left(9,5 \times 10^{6} \mathrm{~kg} / \mathrm{m}^{2}\right)$, algunas de ellas fabricadas con aserrín y almidón utilizando prensas hidráulicas (Tingo, 2012; Martínez, 2015). Tomando en cuenta que en el presente trabajo se empleó la presión generada por la fuerza del brazo humano, se puede considerar que se obtuvo un resultado bastante bueno. Por otra parte y apoyando lo antes mencionado, en un trabajo realizado por Rivarola, Rojo y Arena (2005) sobre la elaboración de placas en base a aserrín y polímeros reciclados, como material alternativo para la industria del mueble, se reportan RA entre $3,0 \times 10^{2} \mathrm{~kg} / \mathrm{m}^{2}$ y $2,1 \times 10^{2} \mathrm{~kg} / \mathrm{m}^{2}$, calificando este resultado como bueno. Así mismo, si se toma en cuenta que la RA esperada para un tablero de partículas convencional está en el orden de $10^{6} \mathrm{~kg} / \mathrm{m}^{2}$ (Peraza et al., 2004), se podría decir que el procedimiento desarrollado genera buenas expectativas para la fabricación de un material alternativo con buenas características de resistencia y biodegradable.

Por otra parte, es importante mencionar que normalmente se espera una correlación directa entre densidad y RA (Tirado, 2015), sin embargo, en este caso la RA del lote A fue dos órdenes de magnitud mayor a la del lote B, a pesar de que la densidad del último es $13 \%$ mayor (Tabla 3). Esto se debe a que las briquetas del lote B, tienen menor cantidad de aserrín que es lo que confiere soporte estructural a la briqueta y además de tener espacios vacíos en su interior (Figura 3), confirmando así las observaciones expresadas en párrafos anteriores.

Es bien conocida la utilidad de la madera y algunos de sus derivados, como aislantes térmicos comúnmente empleados por la industria de la construcción. La conductividad térmica de la madera es baja ubicándose alrededor de $0,13 \mathrm{~W} / \mathrm{mK}$, para la maderas y contrachapados con una densidad alrededor de $0,500 \mathrm{~g} / \mathrm{ml}$; la densidad del pino caribe, que es la fuente de nuestra materia prima, es alrededor de 0,564 g/ml (Arroyo, 2003; Peraza et al., 2004; Celano, Jacobo y Pereira, 2006). Debido a que el componente mayoritario de las briquetas es aserrín y tienen una densidad inferior a la del pino caribe (Tabla 3 ), se infiere que podrían tener buenas cualidades para ser empleadas como material aislante.

En este trabajo no se determinó la conductividad térmica de las briquetas, pero se realizó una estimación de su capacidad de aislamiento térmico (Figuras 1 y 2). Los resultados obtenidos indican que este material es capaz de mantener una temperatura entre $29 \%$ y $38 \%$ por debajo de la fuente de calor a la que fueron expuestas $\left(41,6^{\circ} \mathrm{C}\right.$ y $57,3^{\circ} \mathrm{C}$, respectivamente), por cada grado de incremento en el control 
la briqueta sólo aumentó en $0,32{ }^{\circ} \mathrm{C}$. Esta evidencia sugiere que el rendimiento del material para oponerse al paso del calor es mayor cuanto mayor es la diferencia de temperatura entre la fuente y el ambiente. Las observaciones realizadas, coinciden con lo reportado en otros trabajos que utilizaron residuos de aserrín y cáscara de arroz, aglutinados con almidón de maíz y otras sustancias, con la finalidad de obtener materiales aislantes y al mismo tiempo dar mayor valor a residuos cuyo destino final generalmente es producir energía o ser quemados al aire (Gutiérrez y González, 2012; Gutiérrez, Cadena y Bula, 2014; Medina et al., 2016). A pesar de ello, la evidencia indica que la madera de pino caribe es más eficiente en oponerse al paso del calor que el material obtenido, la diferencia es pequeña aunque significativa alcanzando sólo el $10 \%$ en el peor de los casos. En general, se podría decir que el material obtenido presenta un comportamiento térmico comparable al de la madera de pino caribe, pero que es susceptible a ser mejorado haciendo modificaciones a la metodología y probando otras formulaciones.

Este trabajo constituye el primer paso en una propuesta de aprovechamiento de los residuos de aserrín y una aplicación que podría incrementar su valor agregado en la búsqueda de una mayor sostenibilidad. Finalmente, es necesario acotar que para trabajos posteriores se hace necesario realizar una caracterización térmica completa de las briquetas, así como la determinación de otros parámetros inherentes a la aplicación propuesta. Entre estos se podría nombrar la determinación de la conductividad térmica, susceptibilidad al ataque microbiológico, propiedades ignífugas y su comportamiento frente a condiciones extremas de humedad.

\section{CONCLUSIONES}

Se desarrolló una metodología que podría constituir una opción en el aprovechamiento sostenible de residuos de aserrín provenientes de la transformación de madera de pino caribe, presentando una alternativa a su uso como biocombustible. Las propiedades físico-mecánicas, físico-químicas y el comportamiento térmico del material obtenido indican que podría tener utilidad como aislante térmico, siendo necesaria una mayor caracterización del producto y optimización del proceso, después de lo cual sería pertinente realizar un estudio de factibilidad económica.

Los resultados obtenidos demuestran que los residuos de aserrín, en muchos casos considerado como desecho, podrían ser utilizados como materia prima para el desarrollo de materiales alternativos. Además de la aplicación propuesta en el presente trabajo, sería interesante estudiar las características acústicas del material, lo cual podría resultar en una opción ambientalmente más viable a la utilización del corcho como aislante acústico.

Finalmente, la investigación e implementación de procesos de este tipo cumplirían con la premisa de retardar la devolución al ambiente del $\mathrm{CO}_{2}$ secuestrado en la madera, todo ello sustentado en de los preceptos de la Química e Ingeniería Verde, contribuyendo a la disminución de la huella de carbono generada por la industria forestal y en general, a la mitigación del cambio climático. 


\section{AGRADECIMIENTO}

Expresamos nuestro agradecimiento a la Sección de Ensayos y de Pulpa y Papel del LNPF-ULA, por su colaboración y aportes en la realización del trabajo. A la Sección de Bioenergía, en las personas de la Prof. Adriana Padilla e Ing.MSc. Enid Marcano de Mohali. Al Vicerrectorado Administrativo de la ULA, por la ayuda económica prestada a la Ing. Leixi Viviana García Escalona para la culminación de este trabajo.

\section{REFERENCIAS}

Agama, E., Juárez, E., Evangelista, S., Rosales, O. y Bello, L. (2013). Características del almidón de maíz y relación con las enzimas de su biosíntesis. Agrociencia ,47(1), 01-12.

ANMAT (2003). Administración Nacional de Medicamentos, Alimentos y Tecnología Médica N.5358/12. Ministerio de Salud, República Argentina. Farmacopea Argentina 7a ed. Vol. I. Buenos Aires. Recuperado de: http://www.anmat.gov.ar/webanmat/fna/pfds/ Libro_Primero.pdf.

Arroyo, J. (2003). Propiedades físico mecánicas de la madera (2da ed.). Universidad de Los Andes, Facultad de Ciencias Forestales y Ambientales. Mérida, Venezuela: Taller de publicaciones de la facultad de Ciencias Forestales y Ambientales, 30-48.

Avilés, C. y López, M. (2015). Innovación medioambiental en las empresas del sector forestal. Revista de Responsabilidad Social de la Empresa, (19), 113-132.

Benítez, P. y Gutiérrez, I. (2012). Determinación de contenido de humedad en astillas de Pinus caribaea var. hondurensis, utilizando un horno doméstico de microondas. Ingeniería, 22(1), 17-24.

Celano, J., Jacobo, G. y Pereyra O. (2006). Desarrollo de paneles termo-acústicos a base de residuos de madera para el mercado de la construcción. Universidad Nacional Del Nordeste: Provincia del Chaco-Argentina. Recuperado de: http://www.unne.edu.ar/unnevieja/Web/ cyt/cyt2006/07-Tecnologicas/2006-T-080.pdf.

Concepción, R., Chonillo, R., Lorenzo, A. y Morales, S. (2016). Determinación de las potencialidades de aserrín en la ciudad de Guayaquil como materia prima para la producción de diversos surtidos en la industria forestal. Holos, (4), 105-114.

Food and Agriculture Organization of the United Nations (2004). Terminología unificada sobre bioenergía. Recuperado de: http://www.fao.org/docrep/009/j6439s/j6439s00.htm

Food and Agriculture Organization of the United Nations (2014). Bioenergía y seguridad alimentaria evaluación rápida (BEFS RA). Recuperado de: http://www.fao.org/3/a-bp845s.pdf.

Food and Agriculture Organization of the United Nations (2017). Aprovechamiento potencial de los residuos de la madera para la producción de energía. Recuperado de: http://www. fao.org/docrep/t0269s/t0269S10.htm.

Fonseca, E. y Tierra, L. (2011). Desarrollo de un proceso tecnológico para la obtención de briquetas de aserrín de madera y cascarilla de arroz, y pruebas de producción de gas pobre. (Tesis de pregrado). Escuela Superior Politécnica de Chimborazo, Riobamba, Ecuador. Recuperado de: http://dspace.espoch.edu.ec/handle/123456789/1883. 
García, F. y Dobado, J. (2008). Química sostenible: una alternativa creíble. Anales de la Real Sociedad Española de Química, 104(3), 205-210.

Gallipoliti, V., Martina, P., Corace, J., Aeberhardt, R. y García, E. (2012). Fabricación de briquetas con aserrín blanco de pino. Análisis inmediato y obtención de su poder calorífico. Avances en Energía Renovable y Medio Ambiente, 6, 35-40. Recuperado de: http://www.cricyt.edu. ar/asades/modulos/averma/trabajos/2012/2012-t006-a004.pdf.

Gómez, J. (2008). Ingeniería verde: 12 principios para la sostenibilidad. Ingeniería Química, $458,168-175$.

Greaves, E., Marin, Y., Visaez, F. y Hernández, J. (2015). Forestry plantations of Pinus caribaea in Venezuela as a solar energy collector. Interciencia, 40(7), 457-464.

Gutiérrez, J. y González, A. (2012). Determinación experimental de conductividad térmica de materiales aislantes naturales y de reciclado. Avances en Energías Renovables y Medio Ambiente, 16(8), 41-48. Recuperado de: http://www.cricyt.edu.ar/asades/modulos/averma/ trabajos/2012/2012-t008-a029.pdf.

Gutiérrez, J., Cadena, C. y Bula, A. (2014). Aislamiento térmico producido a partir de cascarilla de arroz aglomerada utilizando almidón producido con saccharomyces cerevisiae. DYNA, 81(184), 1-10. Recuperado de: https://revistas.unal.edu.co/index.php/dyna/rt/ printerFriendly/37679/53957.

Hang, S., Castán, E., Negro, G., Daghero, A., Buffa, E., Ringuelet, A., Satti, P. y Mazzarino, M. (2015). Compostaje de estiércol de feedlot con aserrín/viruta: características del proceso y del producto final. Agriscientia, 32(1), 55-65.

Intergovernmental Panel on Climate Change (2015). Cambio Climático 2014, Mitigación del cambio climático, Resumen para responsables de políticas y resumen técnico. Contribución del grupo de trabajo III al quinto informe de evaluación del Grupo Intergubernamental de Expertos sobre el Cambio Climático. OMM-PNUMA. Editado por Edenhofer y colaboradores. Recuperado de: https://www.ipcc.ch/pdf/assessment-report/ar5/wg3/ WGIIIAR5_SPM_TS_Volume_es.pdf.

López, J., Cajina, C., Ramírez, J., Reyes, E., Olivas, N. y Molina, L. (2016). Evaluación de los parámetros físicos y químicos de las briquetas obtenidas con la máquina briqueteadora construida en FAREM-Estelí. Revista Científica de FAREM-Estelí, 4(16), 3-14.

Loreto, A., Molina, R., Vivas, V., Lugo, A. y Conti, A. (2000). La madera: Una línea de investigación. Tecnología y Construcción, 16(3), 9-20.

Lucas, A., Peso, C., Rodríguez, E. y Prieto, P. (2012). Biomasa, biocombustibles y sostenibilidad. Editor: Centro Tecnológico Agrario y Agroalimentario. ITAGRA.CT. ISBN: 97884-931891-5-0. Recuperado de: http://sostenible.palencia.uva.es/publicaciones.

Martínez, L. (2015). Determinación de las propiedades fisicoquímicas y mecánicas de briquetas elaboradas con aserrín, desechos sólidos, vacasa y tetrabrik, utilizando almidón y cal como aglutinantes. (Tesis de pregrado). Universidad de San Carlos de Guatemala, Guatemala. Recuperado de: http://www.repositorio.usac.edu.gt/3256/1/Ana\%20Luc\%C3\%ADa\%20 Mart\%C3\%ADnez\%20Maldonado.pdf. 
Medina, D., Palma, A. y Castro, H. (2016). Uso de la biomasa extraída de los subproductos del arroz como aislante térmico. Actas de Ingeniería, 2, 39-45.

Organización de las Naciones Unidas (2010). Desarrollo Sostenible, Antecedentes. Asamblea General de las Naciones Unidas, Presidente del $65^{\circ}$ Período de Sesiones. Recuperado de: http://www.un.org/es/ga/president/65/issues/sustdev.shtml.

Ordóñez, O. (2015). Evaluación de la influencia de la formulación de combustibles sólidos densificados de aserrín de pino blanco (Pinus pseudostrobus Lindl), reciclado de carbón activado y almidón de yuca (Manihot esculenta), sobre las propiedades fisicoquímicas y fisicomecánicas de los combustibles sólidos densificados en general. (Tesis de pregrado). Universidad de San Carlos de Guatemala, Guatemala. Recuperado de: http://biblioteca. ingenieria.usac.edu.gt/.

Peraza, F., Arriaga, F. y Peraza, J. (2004). Tableros de madera de uso estructural. Editado por la Asociación de Investigación Técnica de las Industrias de la Madera (AITIM), Madrid España. Recuperado de: https://www.cic.cn.umich.mx/index.php/cn/article/view/330/177.

Pineda, P., Coral, D., Arciniegas, M., Rorales, A. y Rodríguez, M. (2010). Role ofwater in maize starch gelatinization: an study by Differential Scanning Calorimetry. Ingeniería y Ciencia, 6(11), 129-141.

Posso, F. (2004). Estudio del desarrollo de las energías alternativas en Venezuela. Anales de la Universidad Metropolitana, 4(1), 147-164.

Posso, F. y Zambrano, J. (2014). Estimation of electrolytic hydrogen production potential in Venezuela from renewable energies. International Journal of Hydrogen Energy, 39(23), 11846-11853.

Reyes, E., Molina, M., Valero, S., Molina, Y. y Betancourt, R. (2012). Propiedades físicas de la madera de Pinus caribaea var. hondurensis, resinado y no resinado de las plantaciones de Uverito (Monagas, Venezuela). Revista Forestal Venezolana, 56(1), 21-28.

Rivarola, A., Rojo, L. y Arena, A. (2005). Uso de materiales recuperados para la conformación de placas de aglomerado utilizados en la industria del mueble. Avances en Energías Renovables y Medio Ambiente, 9, 19-24.

Rojo, G., Jasso, J. y Velásquez, A. (2003). Las masas forestales como sumideros de $\mathrm{CO}^{2}$ ante un cambio climático global. Revista Chapingo Series Ciencias Forestales y Ambiente, 9(1), 57-67.

Smith, S. y Bracho, Y. (2011). Aprovechamiento de la biomasa residual seca, una alternativa para la generación local de energía eléctrica en Venezuela. Revista Forestal Latinoamericana, 26(1), 25-42.

Soto, G. y Núñez, M. (2008). Fabricación de pellets de carbonilla, usando aserrín de Pinus radiata (D. Don), como material aglomerante. Madera, Ciencia y Tecnología, 10(2), 129-137.

Tingo, M. (2012). Transformación y uso sostenible de los residuos maderables y agrícolas en briquetas en Leoncio Prado-Perú. (Tesis de pregrado). Universidad Nacional Agraria de la Selva, Perú. Recuperado de: http://repositorio.unas.edu.pe/bitstream/handle/UNAS/930/T. EPG-56.pdf?sequence=1\&isAllowed=y 
Tirado, P. (2015). Estudio de la compactación de la cáscara de nuez para mejorar la calidad de briquetas de biomasa (Tesis de pregrado). Universidad Técnica de Ambato, Ambato, Ecuador. Recuperado de: http://repo.uta.edu.ec/handle/123456789/10366 .

Valderrama, A., Curo, H., Quispe, C., Llanto, V. y Gallo, J. (2007). Briquetas de residuos sólidos como fuente de energía en cocinas no convencionales. Revista de Investigación CEDIT, 2, 24-36.

Velázquez, B. (2006). Situación de los sistemas de aprovechamiento de los residuos forestales para su utilización energética. Ecosistemas, 15(1), 77-86.

Vera, A. (2014). Diseño de briquetas ecológicas para la generación de energía calórica y mejoramiento de ecosistemas en el corregimiento de Nebusimake, Municipio de Pueblo Bello-Cesar. (Tesis de pregrado). Universidad Nacional Abierta y a Distancia, Valledupar, Colombia. Recuperado de: http://hdl.handle.net/10596/6111. 